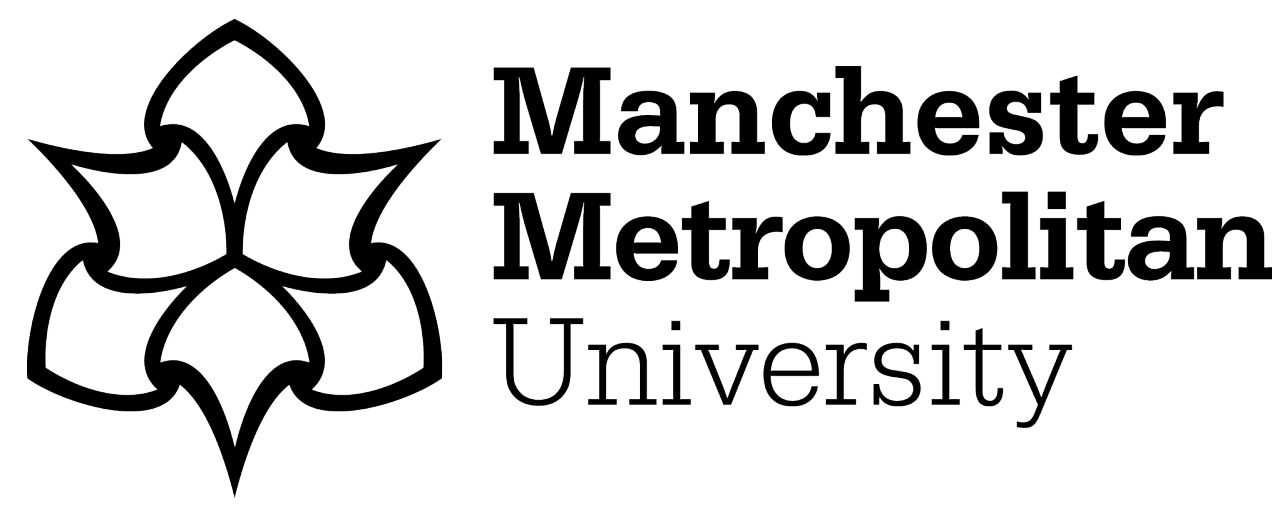

Hogarth, L, Payton, C, Van de Vliet, P, Connick, M and Burkett, B (2018) A novel method to guide classification of para swimmers with limb deficiency. Scandinavian Journal of Medicine and Science in Sports, 28 (11). pp. 23972406. ISSN 0905-7188

Downloaded from: https://e-space.mmu.ac.uk/620802/

Version: Accepted Version

Publisher: Wiley

DOI: https://doi.org/10.1111/sms.13229

Please cite the published version 
DR LUKE HOGARTH (Orcid ID : 0000-0001-7085-4501)

Article type : Original Article

\section{A NOVEL METHOD TO GUIDE CLASSIFICATION OF PARA SWIMMERS WITH LIMB DEFICIENCY}

Luke Hogarth $^{1}$, Carl Payton ${ }^{2}$, Peter Van de Vliet ${ }^{3}$, Mark Connick ${ }^{4}$, Brendan Burkett ${ }^{1}$

${ }^{1}$ School of Health and Sport Sciences, University of the Sunshine Coast, Sippy Downs, Australia.

${ }^{2}$ HEAL Research Centre, Manchester Metropolitan University, Crewe, United Kingdom.

${ }^{3}$ Medical and Scientific Department, International Paralympic Committee, Bonn, Germany.

${ }^{4}$ School of Human Movement Studies, University of Queensland, St Lucia, Queensland, Australia.

Running head: Para swimming classification

Key words: Paralympic, freestyle, disability, partial least squares, machine learning

Corresponding Author:

Luke Hogarth

School of Health and Sport Sciences, University of the Sunshine Coast, Sippy Downs, Queensland, Australia

Postal address: Locked Bag 4, Maroochydore DC QLD 4558

Telephone: +61 754565065

Email: Ihogarth@usc.edu.au

This article has been accepted for publication and undergone full peer review but has not been through the copyediting, typesetting, pagination and proofreading process, which may lead to differences between this version and the Version of Record. Please cite this article as doi: $10.1111 / \mathrm{sms} .13229$

This article is protected by copyright. All rights reserved. 


\section{ABSTRACT}

The International Paralympic Committee has directed International Federations that govern Para sports to develop evidence-based classification systems. This study defined the impact of limb deficiency impairment on $100 \mathrm{~m}$ freestyle performance to guide an evidence-based classification system in Para Swimming, which will be implemented following the 2020 Tokyo Paralympic games. Impairment data and competitive race performances of 90 international swimmers with limb deficiency were collected. Ensemble partial least squares regression established the relationship between relative limb length measures and competitive $100 \mathrm{~m}$ freestyle performance. The model explained $80 \%$ of the variance in 100 $m$ freestyle performance, and found hand length and forearm length to be the most important predictors of performance. Based on the results of this model, Para swimmers were clustered into four-, five-, six- and seven-class structures using nonparametric kernel density estimations. The validity of these classification structures, and effectiveness against the current classification system, were examined by establishing within-class variations in $100 \mathrm{~m}$ freestyle performance and differences between adjacent classes. The derived classification structures were found to be more effective than current classification based on these criteria. This study provides a novel method that can be used to improve the objectivity and transparency of decision-making in Para sport classification. Expert consensus from experienced coaches, Para swimmers, classifiers and sport science and medicine personnel will benefit the translation of these findings into a revised classification system that is accepted by the Para swimming community.

\section{INTRODUCTION}

Classification systems are used in Para sport to promote the vision of the Paralympic Movement: 'to enable Para athletes to achieve sporting excellence and inspire and excite the world.' Classification systems define who is eligible to compete in Para sports and group athletes into classes to minimise the impact of impairment on the competition outcome. ${ }^{1,2}$ For para swimmers with a physical impairment, a functional classification system is currently used to assess the activity limitation caused by the impairment type, location, and severity, and group athletes into S (Freestyle, Backstroke and Butterfly) and SB (Breaststroke)

This article is protected by copyright. All rights reserved. 
classes. ${ }^{3}$ There are ten S (S1-S10) and nine SB classes (SB1-SB9) with lower class numbers indicating greater activity limitation caused by swimmers' physical impairments. Para swimmers with short stature, limb deficiency, leg length difference, impaired passive range of movement, impaired muscle power, hypertonia, ataxia, and athetosis, are eligible to compete within the same class if their impairments are judged to have a similar impact on swimming performance.

Following the inception of the functional classification system in the 1990's, the effectiveness of the World Para Swimming classification system has often been questioned, as it fails to delineate performance between adjacent classes and disadvantages certain physical impairment types within classes. ${ }^{4-6}$ These concerns may result from difficulties with measurement weighting and aggregation, stemming from a lack of understanding of the impact that physical impairments have on swimming performance. ${ }^{2,7}$ To address these concerns the governing body of Para sport, the International Paralympic Committee (IPC), has mandated the development of evidence-based methods of classification in Para sports. ${ }^{8}$ An evidence-based classification system is one that has a clearly stated purpose, being to minimise the impact of impairment on the outcome of competition, and that the methods used to assign class are based on empirical evidence. ${ }^{2}$ The most fundamental aspect of developing evidence-based methods of classification is the quantification of the relationship between impairment and sports performance. ${ }^{9-13}$

Para swimmers with limb deficiency, resulting from trauma or congenital birth defect, have reduced body surface area that impacts their ability to produce propulsive forces and minimise their resistance in the water. ${ }^{14-16}$ The current classification system uses direct limb length measurements and body segment parameters (in cases of bilateral impairments) to determine the relative length of para-swimmers' affected limb segments and summate a points score used for classification. ${ }^{3}$ Points are allocated for the hand, forearm, upper arm, foot, shank, and thigh based on each limb segments' expected contribution to performance in the swimming strokes of the S and SB classes. Direct limb length measurements have been shown to have acceptable reliability, and allow classifiers to measure limb length using a time efficient and user-friendly procedure requiring basic equipment. ${ }^{17-19}$ However, it is unclear whether the points weighting for limb segment lengths result in fair and equitable classification for Para swimmers with limb deficiency.

This article is protected by copyright. All rights reserved. 
Establishing the association between limb length measurements and swimming performance will allow for an improved scientific basis for the classification of swimmers with limb deficiency. Previous research has found measures of absolute limb length or limb segment ratios to have significant associations with swimming performance in non-disabled swimmers. ${ }^{20-22}$ It is important that these factors are not accounted for during the classification process, otherwise swimmers that have a natural advantage due to limb length characteristics unrelated to their impairment may be disadvantaged by the classification process. Limb length measures that are expressed as a percentage of the estimated 'healthy' limb segment length might allow for a useful impairment measure whilst accounting for the natural variance in anthropometric determinants within a cohort of Para swimmers with limb deficiency.

This study aimed to provide scientific evidence to guide a revised classification system, specifically for Para swimmers with limb deficiency that is due for implementation following the 2020 Tokyo Paralympic games. The objectives were: (i) to establish the relationship between limb deficiency impairment and swimming performance, and (ii) propose a novel method for deriving fair and equitable classification structures for Para swimmers with limb deficiency. It was hypothesised that limb length measures would collectively explain the majority of variance in swim performance, and a scientific method for deriving classification structures would result in larger differences between adjacent classes than the current Para swimming classification method.

\section{METHODS}

\subsection{Study design and participants}

A cross-sectional study design was employed. Measures of limb segment length and a performance measure (personal best $100 \mathrm{~m}$ freestyle race time) were analysed for 90 male Para swimmers registered in the International Paralympic Committee (IPC) Sport Data Management System (SDMS) as having a limb deficiency (Table 1). Data were obtained from the IPC SDMS (https://db.ipc-services.org/sdms) for Para swimmers that had received international swimming classification, were registered as being 'active' (i.e. currently 
competing) at the time of data collection and had previously competed at a Paralympic or World Championship event. Para swimmers were removed from analysis if they had a secondary impairment other than limb deficiency. Data were obtained with permission from World Para Swimming under approved ethical guidelines from the institution's Human Research Ethics Committee (A/16/891).

\subsection{Data}

All data were obtained from athlete classification records listed on the IPC SDMS. Personal best race time for the $100 \mathrm{~m}$ freestyle was recorded for each Para swimmer and expressed as mean race speed $(\mathrm{m} / \mathrm{s})$. Race times that were recorded during short course events were excluded. Para swimmers' limb deficiency impairments were described using broad classifications (e.g. single below elbow) and novel measures of limb length. Absolute limb length measures were obtained from Para swimmers' classification records and converted to relative limb length measures for analysis. Relative limb length measures expressed the remaining length of the limb segment as a percentage of the corresponding 'healthy' limb segment length. In cases where Para swimmers had no corresponding limb segment for comparison, the 'healthy' limb segment length was estimated using body segment ratios that are found in the classification manual. ${ }^{3}$ A total of six limb length measures were calculated for each Para swimmer that described the remaining limb length of the hand, forearm, upper arm, foot, shank and thigh segments, with the left and right limb segments each contributing to half of the score. The body landmarks and procedures used to define these limb segments are outlined in the classification manual. ${ }^{3}$

\subsection{Statistical analyses}

Statistical analyses were carried out using the R Stats package version 3.4.0 (R Core Team, 2017). Normative values (mean and standard deviation) for $100 \mathrm{~m}$ freestyle performance were established for limb deficiency impairment groups. Pearson's correlation coefficients were calculated to establish the absolute strength of association between limb length measures and $100 \mathrm{~m}$ freestyle performance.

This article is protected by copyright. All rights reserved. 


\subsubsection{Ensemble partial least squares regression}

The relationship between limb deficiency impairment and personal best $100 \mathrm{~m}$ freestyle performance was established using an ensemble partial least squares regression analysis. ${ }^{23}$ The partial least squares method transforms the data to reduce both the predictor (independent) and response (dependent) variables to $\mathrm{x}$ - and $\mathrm{y}$-components. The derived $\mathrm{x}$ components are then used to predict the response using regression analysis. The advantage of partial least squares regression is that while the independent variables may be collinear, the derived $\mathrm{x}$-components will be independent of one another while also keeping most of the variance explained in the dataset. ${ }^{24}$

The "enpls" argument in the enpls package ${ }^{25}$ was used to conduct partial least squares regression on 500 Monte Carlo experiments with a sampling ratio of 0.8 . As the determined effects of independent variables to any model are dependent upon the cases included in analyses, ensemble learning methods may improve prediction accuracy and stability of regression models by exploiting the statistical distribution of variable coefficients and prediction errors. ${ }^{25} \mathrm{~K}$-fold cross validation was used to examine the stability of the ensemble partial least squares model using the "cv.enpls()" argument. ${ }^{25}$

Variable coefficients and importance scores were calculated using the "fs.enpls()" function in the enpls package. ${ }^{25}$ The vector of regression coefficients across multiple Monte Carlo experiments were used to indicate predictor importance because it is a single measure of association between the predictor variables and the response. ${ }^{25,26}$ The variable importance score was calculated as the mean of the variable coefficient divided by the standard deviation of the variable coefficient for the 500 Monte Carlo experiments. ${ }^{25}$ Thus, a larger variable importance score indicates a larger and more consistent regression coefficient across the 500 Monte Carlo experiments.

\subsubsection{Cluster analysis via nonparametric density estimation}

To derive new classification structures clusters were identified from the kernel density estimations of 'predicted performance' using the pdfCluster package. Various bandwidths were used to adjust the smoothing parameters and identify local maxima and minima of a four-, five-, six- and seven-class structure. The 'predicted performance' of Para swimmers is 
a measure of estimated activity limitation that does not explain their actual sporting performance or ability, but the level of difficulty that Para swimmers are expected to have for the $100 \mathrm{~m}$ freestyle resulting from their limb deficiency impairment.

\subsubsection{Evaluating the fairness and equity of classification structures}

The existing and new classification structures were evaluated by determining the differences in 'actual performance' between adjacent classes, and the within-class variations in actual and predicted performances. A more effective classification structure was considered to show larger differences in performance between adjacent classes, and smaller within-class variations in actual and predicted performance (i.e. estimated activity limitation). ${ }^{2,27,28}$ The magnitude of group differences between adjacent classes were determined using standardised differences with the precision of estimates indicated with $90 \%$ confidence limits $(C L)$. The magnitude of difference was assessed as trivial $(<0.2)$, small (0.21-0.6), moderate (0.61-1.2), large (1.21-2.0), and very large (>2.0) based on previously standardised criteria. ${ }^{29}$ The difference was reported as unclear when the $90 \% \mathrm{CL}$ crossed the thresholds for both a substantially positive $(0.2)$ and negative $(-0.2)$ value.

\section{RESULTS}

The existing $\mathrm{S}$ classes and personal best $100 \mathrm{~m}$ freestyle performances of Para swimmers included in this study are reported in Table 1. Para swimmers' personal best $100 \mathrm{~m}$ freestyle mean race speed (time) ranged from $0.75 \mathrm{~m} / \mathrm{s}(133.3 \mathrm{~s})$ to $1.84 \mathrm{~m} / \mathrm{s}(54.3 \mathrm{~s})$. The remaining limb lengths of the forearm $(r=0.70, p<0.01)$, hand $(r=0.61, p<0.01)$, upper arm $(r=0.52$, $p<0.01)$, thigh $(r=0.38, p<0.05)$, shank $(r=0.33, p<0.05)$, and foot $(r=0.23, p<0.05)$ were found to have significant correlations with $100 \mathrm{~m}$ freestyle performance.

\subsection{Ensemble partial least squares regression}

A minimum of 2 and maximum of 3 components were extracted from partial least squares analysis for 500 Monte Carlo experiments. The components of separate partial least squares regressions explained between $74.9 \%$ and $83.6 \%$ of the variance in $100 \mathrm{~m}$ freestyle performance, and between $75.0 \%$ and $88.1 \%$ of the variance in limb length variables. The 
ensemble partial least squares regression explained $79.5 \%$ of the variance in $100 \mathrm{~m}$ freestyle performance $\left(R^{2}=0.795, R M S E=0.118\right)$, and 10 -fold cross validation reported similar prediction accuracy and error $\left(R^{2}=0.767\right.$, RMSE $\left.=0.125\right)$ (Figure 1$)$. The most important predictor variable was forearm length, followed by hand length and shank length (Figure 2).

Predicted $100 \mathrm{~m}$ freestyle race speeds ranged from $0.96 \mathrm{~m} / \mathrm{s}(104.2 \mathrm{~s})$ to $1.82 \mathrm{~m} / \mathrm{s}(54.9 \mathrm{~s})$. There were gradual differences in predicted performance for Para swimmers with a single below ankle (range $=1.77$ to $1.82 \mathrm{~m} / \mathrm{s})$, below knee $($ range $=1.67$ to $1.77 \mathrm{~m} / \mathrm{s}$ ) and above knee $($ range $=1.47$ to $1.65 \mathrm{~m} / \mathrm{s}$ ) limb deficiency impairment, as well as for Para swimmers with a single below wrist (range $=1.74$ to $1.78 \mathrm{~m} / \mathrm{s}$ ), below elbow (range $=1.61$ to $1.73 \mathrm{~m} / \mathrm{s}$ ) and above elbow (range $=1.48$ to $1.53 \mathrm{~m} / \mathrm{s}$ ) impairment. Para swimmers with limb deficiency affecting two or more limbs showed large variations in predicted performance (Figure 3A). All types of limb deficiency impairment showed similar distributions in residuals (Figure 3B), and no limb deficiency group reported a significant difference between actual and predicted $100 \mathrm{~m}$ freestyle performance.

\subsection{Validity of classification structures}

There was considerable overlap in the predicted performances of Para swimmers in their current S class, particularly between classes S4 and S5 and S5 and S6 (Table 2). Although other classes showed larger differences with their adjacent classes, there were cases of predicted performance overlapping for every adjacent class. Cluster analyses using kernel density estimations with variable smoothing parameters (bandwidths $=0.02$ to 0.04 ) identified cluster centres and cut-points in the distribution of predicted $100 \mathrm{~m}$ freestyle performance to derive a four-, five-, six- and seven-class structure (Figure 4). The four( $\mathrm{ES} \pm 90 \% \mathrm{CL}=1.2 \pm 0.7$ to $2.1 \pm 0.5)$, five- $(\mathrm{ES} \pm 90 \% \mathrm{CL}=0.9 \pm 0.6$ to $1.5 \pm 0.5)$ and six-class $(\mathrm{ES} \pm 90 \% \mathrm{CL}=0.8 \pm 0.8$ to $1.6 \pm 0.7$ ) structures showed consistently larger differences in actual performance between adjacent clusters than for the current classification system (ES $\pm 90 \%$ $C L=0.5 \pm 0.9$ to $1.4 \pm 0.6$ ) (Table 2 ). The seven-class system showed an unclear difference between cluster 2 and $3(\mathrm{ES} \pm 90 \% \mathrm{CL}=0.2 \pm 1.0)$.

This article is protected by copyright. All rights reserved. 


\section{DISCUSSION}

The IPC has mandated that research be conducted to guide the development of evidencebased classification systems in Para sport. ${ }^{3}$ The most fundamental aspect of developing evidence-based classification systems is the quantification of the relationship between impairment and sports performance. ${ }^{1,2}$ This study used ensemble modelling to establish the relationship between limb deficiency impairment and Para swimming performance, and to provide a one-dimensional estimate of activity limitation that was used to develop classification structures and allocate class. The following sections will discuss the efficacy of this classification method, which is partial to the accuracy of the prediction model, the fairness of classification structures that are derived from the model, and how the classification method might be perceived by relevant stakeholders in Para swimming.

\subsection{Modelling the impact of limb deficiency impairment on performance}

Limb segment length measures explained $80 \%$ of the variance in $100 \mathrm{~m}$ freestyle performance in Para swimmers with limb deficiency $\left(R^{2}=0.80\right.$, RMSE $\left.=0.12\right)$. The amount of variance in performance that should be explained by physical impairment is difficult to determine, as a certain amount of variance will be explained by technical, physiological and psychological attributes that shouldn't account for Para swimmers' classification. In another study, measures of arm and trunk impairment explained between $23 \%$ and $37 \%$ of the total variance in performance tests in wheelchair rugby and basketball athletes. ${ }^{13}$ The results of this study on international-standard Para swimmers are promising, particularly as the partial least squares regression showed relatively consistent prediction accuracies across multiple Monte Carlo experiments $\left(R^{2}=0.75-0.84\right)$ and cross-validation reported similar prediction accuracy and error to the partial least squares regression $\left(R_{c v}^{2}=0.77, R_{M S E}=0.13\right)$. These results indicate that the ensemble partial least squares regression model has sufficient accuracy and stability, suggesting it may provide an accurate estimate of activity limitation resulting from Para swimmers' limb deficiency impairments.

When examining the prediction accuracy of the ensemble partial least squares regression, it appears that the model overestimates the performance of Para swimmers with personal best $100 \mathrm{~m}$ freestyle performances $\leq 1.15 \mathrm{~m} / \mathrm{s}$ (Figure 1). These Para swimmers often use modified swimming strokes in freestyle events due to the severity of their impairment,

This article is protected by copyright. All rights reserved. 
rather than swimming front crawl as more typical in the higher sport classes. Therefore, limb segment lengths may have varied contributions to the performance of these Para swimmers depending on their unique stroke pattern. It is also possible that the impact of impairment severity on sports participation and training progression might result in lower competitive standards in the classes in which these Para swimmers compete. For instance, Para swimmers with severe impairments need greater assistance in accessing suitable training facilities and have considerably less training volume than Para swimmers in higher classes, which could stunt their training progression. ${ }^{30}$ Better prediction accuracies for these Para swimmers might be reported once participation rates and competitive standards in the lower sport classes improve with continued progression of the Paralympic Movement.

The interpretation of the partial least squares regression and whether it is perceived to be 'correct' by coaches, athletes and spectators is an important consideration if it is to be used to allocate class. The results indicated forearm length, hand length, and shank length to be the most important predictors of $100 \mathrm{~m}$ freestyle performance (Figure 2). These results are partially supported by correlation coefficients between limb length variables and $100 \mathrm{~m}$ freestyle performance, that showed forearm length $(r=0.70, p<0.01)$ and hand length $(r=0.61, p<0.01)$ to have the strongest correlations with performance. The upper limbs contribute to the majority of propulsive force during tethered front crawl swimming, ${ }^{31}$ and the hand and forearm segment have been shown to produce greater hydrodynamic forces than the upper arm due to greater angular and linear velocities during the swim stroke. ${ }^{32,33}$ Interestingly, upper arm length had a stronger correlation $(r=0.52, p<0.01)$ with $100 \mathrm{~m}$ freestyle performance than all the lower-body limb segment lengths $(r=0.23$ to 0.38 , $p<0.03$ ), despite upper arm length being the least important predictor variable (Figure 2). These results are likely explained by high collinearity between the upper-and lower-body limb segments that is accounted for by the transformation of predictor variables into latent structures during partial least squares analysis. Indeed, research has suggested that the upper arm segment might even contribute to net resistive drag forces during front crawl swimming, as greater angular velocities of the upper arm segment are required to create positive net propulsive force with proportionate increases in swimming speed. ${ }^{16,34,35}$ These findings emphasise the importance of classification methods being based on research that uses multivariate statistical models to establish the relative contribution of impairment

This article is protected by copyright. All rights reserved. 
measures to sports performance, rather than absolute associations that have been established in isolation.

It is interesting to note that forearm length was found to be a more important predictor of $100 \mathrm{~m}$ freestyle performance than hand length (Figure 2). According to the partial least squares regression, a single through elbow impairment $(1.59 \mathrm{~m} / \mathrm{s}, 86.1 \%)$ is estimated to have an additional $7.6 \%$ decrement in performance than a single through wrist impairment $(1.74 \mathrm{~m} / \mathrm{s}, 93.8 \%)$ compared with the estimated performance of an able-bodied swimmer $(1.85 \mathrm{~m} / \mathrm{s})$. These results are counter-intuitive to research showing the hand segment to create considerably larger hydrodynamic forces during front crawl swimming than the forearm segment. ${ }^{33}$ The hand has advantage in terms of linear velocities and swimmers use minor hand movements at the wrist joint so that the hand is in the most efficient position possible relative to the other upper arm segments. ${ }^{32,33,36}$ There are several explanations for the results of our study. First, while there may be greater hydrodynamic forces reported at given sites for the hand segment, the forearm segment might contribute greater net positive propulsive force than the hand due to having greater propelling surface area. ${ }^{33,37}$ Further, the relative contribution of the forearm segment to $100 \mathrm{~m}$ freestyle performance may increase for Para swimmers with an upper limb impairment, as they show varied stroke patterns to increase the angular and linear velocities of their 'intact' limb segments and maximise their net propulsive force during swimming. ${ }^{16,38,39}$ Second, this study only included Para swimmers in the analyses, of which the least impaired Para swimmers with an upper limb impairment would have up to $\sim 50 \%$ of their hand length intact as per the minimum impairment eligibility criteria. ${ }^{3}$ It is possible that the contribution of hand length to $100 \mathrm{~m}$ freestyle performance was underestimated by the partial least squares regression, as the results are dependent upon the cases included in the analysis that explain the variance within the dataset.

\subsection{Validity of classification structures}

The second objective of this study was to derive new classification structures for Para swimmers based on the estimated activity limitation resulting from their limb deficiency impairment. Kernel density estimations of Para swimmers 'predicted performance' were used to derive a four-, five-, six- and seven-class structure (Figure 4). The validity of these 
structures was evaluated by establishing the within-class variation in estimated activity limitation and differences in actual performance between adjacent classes. ${ }^{27,28}$ Based on these criteria, the newly derived classification structures were found to be fairer and more equitable than the current classification system as the classification method: (i) better accounts for the impact that limb deficiency impairment has on Para swimming performance, (ii) results in classes that have sequential and clear differences in $100 \mathrm{~m}$ freestyle performance, and (iii) allocates class cut-points along the spectrum of activity limitation where there is less chance of Para swimmers being allocated to the wrong class.

In this study, the current classification system was shown to have unclear differences in 100 $\mathrm{m}$ freestyle performance between existing classes S7 and S6 and S6 and S5 (Table 2). Two or more of these classes could be collapsed to provide a fairer classification system. However, there was also considerable overlap in the predicted performance of Para swimmers between all adjacent classes based on the results of the partial least squares regression. This suggests that in all classes of the current classification system, there are swimmers that are advantaged or disadvantaged by the location and severity of their limb deficiency impairment.

There are two limitations of the current classification method that might contribute to this problem. First, the points allocated to different limb segments do not equate to a summative point score that accurately defines the activity limitation of Para swimmers for the $100 \mathrm{~m}$ freestyle. ${ }^{3}$ Second, the S class is used to structure competition for all event distances of the freestyle, butterfly and backstroke swim strokes, not just the $100 \mathrm{~m}$ freestyle. The relative importance of limb segment lengths might change for different swim strokes and event distances, as do other important variables including stroke frequency and length, temporal coordination of propulsive actions, upper-limb and lower-limb muscle activity and coordination, and the contribution of race segments to overall performance..$^{40-43}$ Methods that better account for the influence of swim stroke and event distance on the activity limitation resulting from limb deficiency impairments might provide fairer and more equitable classification structures.

This article is protected by copyright. All rights reserved. 
The valid classification structures that were derived from Para swimmers' predicted performances is further evidence that the ensemble partial least squares regression better estimates the activity limitation resulting from limb deficiency impairment than current classification methods. The four-, five- and six-class structures all showed adjacent clusters had moderate to large differences in actual $100 \mathrm{~m}$ freestyle performance (Table 2). The seven-class structure showed an unclear difference between clusters 2 and 3, suggesting that a classification structure with no more than six classes will provide fair and equitable competition. Deciding whether a four-, five- or six-class structure should be used for classification of these swimmers is a more challenging task.

The IPC position stand ${ }^{2}$ does not define what the difference in performance between classes should be, other than 'within any given class, the range of activity limitation should never be so large that athletes with impairments causing the greatest activity limitation are significantly disadvantaged when competing against those with impairments causing the least activity limitation.' Experimental research that investigates the sport-specific determinants of performance in Para swimmers within these classes might provide further insight into the optimal number of classes and their cut-points. Regardless, these results demonstrate the potential of using ensemble modelling to estimate the activity limitation resulting from physical impairment and to improve the objectivity and transparency of decision-making in Para classification.

An advantage of the classification method developed in this study is that a one-dimensional estimate of activity limitation can be calculated to provide a direct comparison of the impact that various limb deficiency impairments have on sport performance. These comparisons are meaningful as they report the results of classification in a real-life context that provide key stakeholders (i.e. Para swimmers, coaches, classifiers, sport science personnel and administrators) with an understanding of the scientific evidence underpinning class allocation and structure. In this study, Para swimmers with diverse types of limb deficiency (i.e. location) were grouped into the same classes based on their estimated activity limitation, with class cut-points established along the spectrum of activity limitation where there were relatively fewer Para swimmers. This is advantageous for structuring competition in Para Swimming that includes Para athletes with diverse types and severity of physical impairment. Further, establishing class cut-points in this way minimises the risk of

This article is protected by copyright. All rights reserved. 
Para swimmers being allocated to the wrong class because they are situated close to the class cut-point.

\subsection{Study limitations and future research directions}

This study was limited to the $100 \mathrm{~m}$ freestyle in a male-only cohort of Para swimmers. This was intentional as the statistical modelling was complex and a single sample of 90 swimmers of international-standard was considered the most appropriate approach. Future studies could apply these methods to different swimming events as it has been postulated that swim stroke and event distance might influence the relationship between physical impairment and performance. ${ }^{4,6}$ Such studies might have important implications for the structure of classification systems in Para swimming; in particular, whether classification should account for any influence of swim stroke or event distance on the impact that physical impairment has on performance. Further, although sex is unlikely to influence the impact of limb deficiency on Para swimming performance, research is warranted to determine the relationships between limb length measures and performance in female cohorts.

The difficulty in lay interpretation of this study's results might limit the impact it has on a revised classification system. It is important that the results of this study are corroborated by further research. The contribution of the limb segments to swim performance is complex, as the upper and lower limb extremities have been shown to have non-linear contributions to swim velocity during maximal arms-only, legs-only and full stroke swimming in ablebodied participants. ${ }^{44}$ These contributions are likely to be more complex based on the variations in impairment severity and location that occur in a Para swimming population. Experimental studies that investigate the impact of limb deficiency impairment on instantaneous velocity, propulsion and drag profiles of Para swimmers might evidence the relative contribution of different limb segments to Para swimming performance. Further, studies should investigate measures that might better describe the severity and distribution of impairment that are associated with activity limitation, such as surface area and asymmetry measurements.

This article is protected by copyright. All rights reserved. 
Despite the advantages of this study's methods, it's important to note that there are numerous statistical algorithms that could be used to derive valid classification structures in Para sport. Each algorithm would result in a different classification structure with its own advantages and disadvantages, although how classification systems are perceived and interpreted by internal (e.g. Para athletes, coaches) and external (e.g. media, spectators) stakeholders ultimately influences their effectiveness in promoting the vision of the Paralympic Movement. Expert consensus by experienced coaches, Para swimmers, classifiers, sport science personnel and administrators is important to validate this study's findings so that they can be translated into a revised classification system that is accepted by key members of the Para Swimming community.

\section{PERSPECTIVE}

This study's findings can help guide a revised classification system due for implementation following the 2020 Tokyo Paralympic games. Ensemble partial least squares regression was shown to provide accurate predictions on the activity limitation experienced by Para swimmers with limb deficiency for the $100 \mathrm{~m}$ freestyle. Based on these predictions, fair and equitable classification structures were derived that showed clearer and more consistent differences in $100 \mathrm{~m}$ freestyle performance between adjacent classes than for existing classification. The translation of this study's findings into an effective classification system that is accepted by the Para swimming community will be aided by further research that (i) examines the influence of swim stroke and event distance on the impact of limb deficiency on Para swimming performance, (ii) investigates the relationships between limb deficiency impairment and propulsion and drag profiles of Para swimmers, and (iii) gains expert consensus from experienced coaches, Para swimmers, classifiers, sport science and medicine personnel, and administrators. In addition to guiding revised classification in Para swimming, this study provides a novel scientific method that can be used in other Para sports to improve the objectivity and transparency of decision-making in classification.

This article is protected by copyright. All rights reserved. 


\section{ACKNOWLEDGEMENTS}

This research was supported by the International Paralympic Committee (IPC) and UK Sport. Mark Connick is a member of the IPC Classification Research and Development Centre (Physical Impairments), which is supported by the International Paralympic Committee.

\section{REFERENCES}

1. Tweedy SM, Mann D, Vanlandewijck YC. Research needs for the development of evidence-based systems of classification for physical, vision, and intellectual impairments. In: Vanlandewijck YC, Thompson WR, eds. Training and Coaching the Paralympic Athlete: John Wiley \& Sons, Ltd.; 2016:122-149.

2. Tweedy SM, Vanlandewijck YC. International Paralympic Committee position standbackground and scientific rationale for classification in paralympic sport. Brit J Sport Med. 2009;10:1136-1186.

3. International Paralympic Committee. World Para Swimming Classification Rules and Regulations. International Paralympic Committee; 2017.

4. Daly DJ, Vanlandewijck Y. Some criteria for evaluating the "fairness" of swimming classification. Adapt Phys Act Q. 1999;16(3):271-289.

5. Wu SK, Williams T. Paralympic swimming performance, impairment, and the functional classification system. Adapt Phys Act Q. 1999;16(3):251-270.

6. Burkett B, Payton C, Van De Vliet P, et al. Performance characteristics of para swimmers - how effective is the swimming classification system? Phys Med Rehabil Clin N Am. 2018;29(2):333-346.

7. Tweedy SM, Beckman EM, Connick MJ. Paralympic classification: conceptual basis, current methods, and research update. PM R. 2014;6(8 Suppl):S11-17.

8. International Paralympic Committee. IPC Athlete Classification Code. 2015; https://www.paralympic.org/classification/2015-athlete-classification-code.

9. Hyde A, Hogarth L, Sayers $M$, et al. The Impact of an Assistive Pole, Seat Configuration, and Strength in Paralympic Seated Throwing. Int J Sports Physiol Perform. 2017;12(7):977-983.

This article is protected by copyright. All rights reserved. 
10. Connick MJ, Beckman E, Spathis J, Deuble R, Tweedy SM. How Much Do Range of Movement and Coordination Affect Paralympic Sprint Performance? Med Sci Sports Exerc. 2015;47(10):2216-2223.

11. Beckman EM, Connick MJ, Tweedy SM. How much does lower body strength impact Paralympic running performance? Eur J Sport Sci. 2016;16(6):669-676.

12. Runciman P, Tucker R, Ferreira S, Albertus-Kajee $\mathrm{Y}$, Derman W. Paralympic athletes with cerebral palsy display altered pacing strategies in distance-deceived shuttle running trials. Scand J Med Sci Sports. 2016;26(10):1239-1248.

13. Altmann VC, Groen BE, Hart AL, Vanlandewijck YC, van Limbeek J, Keijsers NLW. The impact of trunk impairment on performance-determining activities in wheelchair rugby. Scand J Med Sci Sports. 2017;27(9):1005-1014.

14. Oh YT, Burkett B, Osborough C, Formosa D, Payton C. London 2012 Paralympic swimming: passive drag and the classification system. $\mathrm{Br} J$ Sports Med. 2013;47(13):838-843.

15. Lee $\mathrm{CJ}$, Sanders RH, Payton CJ. Changes in force production and stroke parameters of trained able-bodied and unilateral arm-amputee female swimmers during a $30 \mathrm{~s}$ tethered front-crawl swim. J Sports Sci. 2014;32(18):1704-1711.

16. Lecrivain G, Slaouti A, Payton C, Kennedy I. Using reverse engineering and computational fluid dynamics to investigate a lower arm amputee swimmer's performance. J Biomech. 2008;41(13):2855-2859.

17. Marfell-Jones MJ, Stewart $A D$, de Ridder JH. International Standards for Anthropometric Assessment. Wellington, New Zealand: The International Society for the Advancement if Kinathropometry; 2012.

18. Carr R, Blade L, Rempel R, Ross WD. Technical note: on the measurement of direct vs. projected anthropometric lengths. Am J Phys Anthropol. 1993;90(4):515-517.

19. Day JAP. Bilateral symmetry and reliability of upper limb measurements. Perspectives in Kinanthropometry. Champaign, IL: Human Kinetics; 1986:257-261.

20. Nevill AM, Oxford SW, Duncan MJ. Optimal Body Size and Limb Length Ratios Associated with 100-m Personal-Best Swim Speeds. Med Sci Sports Exerc. 2015;47(8):1714-1718.

This article is protected by copyright. All rights reserved. 
21. Dingley AA, Pyne DB, Burkett B. Relationships Between Propulsion and Anthropometry in Paralympic Swimmers. Int J Sports Physiol Perform. 2015;10(8):978-985.

22. Chatard JC, Lavoie JM, Lacour JR. Analysis of determinants of swimming economy in front crawl. Eur J Appl Physiol Occup Physiol. 1990;61(1-2):88-92.

23. Schranz N, Tomkinson G, Olds T, Petkov J, Hahn AG. Is three-dimensional anthropometric analysis as good as traditional anthropometric analysis in predicting junior rowing performance? J Sports Sci. 2012;30(12):1241-1248.

24. Mevik $\mathrm{BH}$, Wehrens R. The pls package: Principal component and partial least squares regression in R. J Stat Softw. 2007;18(2):1-23.

25. Cao DS, Deng ZK, Zhu MF, Yao ZJ, Dong J, Zhao RG. Ensemble partial least squares regression for descriptor selection, outlier detection, applicability domain assessment, and ensemble modeling in QSAR/QSPR modeling. J Chemometr. 2017;31(11):e2922.

26. Mehmood T, Liland KH, Snipen L, Saebo S. A review of variable selection methods in Partial Least Squares Regression. Chemometr Intell Lab. 2012;118:62-69.

27. Connick MJ, Beckman E, Vanlandewijck Y, Malone LA, Blomqvist S, Tweedy SM. Cluster analysis of novel isometric strength measures produces a valid and evidencebased classification structure for wheelchair track racing. Br J Sports Med. 2017.

28. Altmann VC, Groen BE, Hart AL, Vanlandewijck YC, Keijsers NLW. Classifying trunk strength impairment according to the activity limitation caused in wheelchair rugby performance. Scand J Med Sci Sports. 2017:n/a-n/a.

29. Hopkins WG. Sample Sizes for Magnitude-Based Inferences about Clinical, Practical or Mechanistic Significance. Med Sci Sport Exer. 2006;38(5):S528-S529.

30. Fulton SK, Pyne DB, Hopkins WG, Burkett B. Training characteristics of paralympic swimmers. J Strength Cond Res. 2010;24(2):471-478.

31. Morouco PG, Marinho DA, Izquierdo M, Neiva H, Marques MC. Relative Contribution of Arms and Legs in $30 \mathrm{~s}$ Fully Tethered Front Crawl Swimming. Biomed Res Int. 2015;2015:563206.

32. Miller DI. Biomechanics of swimming. Exerc Sport Sci Rev. 1975;3:219-248.

33. Vorontsov AR, Rumyantsev VA. Propulsive Forces in Swimming. Biomechanics in Sport: Blackwell Science Ltd; 2008:205-231.

This article is protected by copyright. All rights reserved. 
34. Lecrivain G, Payton C, Slaouti A, Kennedy I. Effect of body roll amplitude and arm rotation speed on propulsion of arm amputee swimmers. J Biomech. 2010;43(6):1111-1117.

35. Hay JG, Thayer AM. Flow visualization of competitive swimming techniques: the tufts method. J Biomech. 1989;22(1):11-19.

36. Toussaint HM, Beek PJ. Biomechanics of competitive front crawl swimming. Sports Med. 1992;13(1):8-24.

37. Cross A, Collard M, Nelson A. Body segment differences in surface area, skin temperature and 3D displacement and the estimation of heat balance during locomotion in hominins. PLoS One. 2008;3(6):e2464.

38. Osborough CD, Payton CJ, Daly DJ. Influence of swimming speed on inter-arm coordination in competitive unilateral arm amputee front crawl swimmers. Hum Mov Sci. 2010;29(6):921-931.

39. Osborough CD, Payton CJ, Daly DJ. Relationships Between the Front Crawl Stroke Parameters of Competitive Unilateral Arm Amputee Swimmers, With Selected Anthropometric Characteristics. J App Biomech. 2009;25(4):304-312.

40. Burkett B, Mellifont R. Sport Science and Coaching in Paralympic Swimming. Int J Sports Sci Coa. 2008;3(1):105-112.

41. Daly DJ, Malone LA, Smith DJ, Vanlandewijck Y, Steadward RD. The contribution of starting, turning, and finishing to total race performance in male paralympic swimmers. Adapt Phys Act Q. 2001;18(3):316-333.

42. Pelayo P, Alberty M, Sidney M, Potdevin F, Dekerle J. Aerobic Potential, Stroke Parameters, and Coordination in Swimming Front-Crawl Performance. Int J Sports Physiol Perform. 2007;2(4):347-359.

43. Ikuta Y, Matsuda Y, Yamada Y, Kida N, Oda S, Moritani T. Relationship between decreased swimming velocity and muscle activity during 200-m front crawl. Eur J Appl Physiol. 2012;112(9):3417-3429.

44. Bartolomeu RF, Costa MJ, Barbosa TM. Contribution of limbs' actions to the four competitive swimming strokes: a nonlinear approach. J Sports Sci. 2018:1-10.

This article is protected by copyright. All rights reserved. 
TABLES

Table 1. Current $\mathrm{S}$ class and personal best $100 \mathrm{~m}$ freestyle performance of Para swimmers with limb deficiency.

\section{$S$ class PB $100 \mathrm{~m}$ freestyle $(\mathrm{m} / \mathrm{s})$}

n Range Mean (SD)

Entire cohort

$\begin{array}{ccc}90 & 4-10 & 1.49(0.26) \\ 16 & 4-8 & 1.17(0.27) \\ 5 & 4-6 & 1.31(0.19) \\ 7 & 7-10 & 1.45(0.19) \\ 10 & 9 & 1.60(0.11) \\ 9 & 9-10 & 1.72(0.08) \\ 2 & 10 & 1.73(0.04) \\ 10 & 4-8 & 1.30(0.22) \\ 10 & 7-8 & 1.48(0.14) \\ 16 & 9 & 1.69(0.10) \\ 5 & 10 & 1.74(0.08)\end{array}$

Three or more limbs

Double, upper and lower limb

Double, above or below knee

Single, above knee

Single, below knee

Single, below ankle

Double, above or below elbow

Single, above elbow

Single, below elbow

Single, below wrist

This article is protected by copyright. All rights reserved. 
Table 2. Normative values, differences and within class variations in $100 \mathrm{~m}$ freestyle performance for the current classification system, and the newly derived class structures.

\begin{tabular}{|c|c|c|c|c|c|c|}
\hline \multirow[b]{2}{*}{$\begin{array}{c}\text { Class } \\
\text { structure }\end{array}$} & \multirow[b]{2}{*}{$\begin{array}{c}\text { Cluster/ } \\
\text { class number }\end{array}$} & \multicolumn{3}{|c|}{ Actual $100 \mathrm{~m}$ freestyle $(\mathrm{m} / \mathrm{s})$} & \multicolumn{2}{|c|}{$\begin{array}{l}\text { Predicted } 100 \mathrm{~m} \\
\text { freestyle }(\mathrm{m} / \mathrm{s})\end{array}$} \\
\hline & & Mean (SD) & CV (\%) & ES (QO) & Mean (SD) & CV (\%) \\
\hline \multirow{7}{*}{$\begin{array}{l}\text { Current } \\
\text { Para } \\
\text { Swimming } \\
\text { method }\end{array}$} & S10 (n=15) & $1.72(0.08)$ & 4.5 & $\bmod$ & $1.74(0.04)$ & 2.5 \\
\hline & S9 $(n=29)$ & $1.66(0.11)$ & 6.7 & la & $1.64(0.07)$ & 4.5 \\
\hline & S8 $(n=15)$ & $1.49(0.13)$ & 8.9 & $\bmod$ & $1.51(0.04)$ & 2.7 \\
\hline & $S 7(n=6)$ & $1.38(0.18)$ & 12.9 & unc & $1.37(0.13)$ & 9.7 \\
\hline & $S 6(n=12)$ & $1.29(0.17)$ & 13.1 & unc & $1.24(0.11)$ & 8.5 \\
\hline & S5 $(n=7)$ & $1.15(0.21)$ & 18.6 & $\bmod$ & $1.15(0.13)$ & 11.1 \\
\hline & $S 4(n=6)$ & $0.97(0.15)$ & 15.4 & & $1.08(0.11)$ & 10.6 \\
\hline \multirow{4}{*}{$\begin{array}{c}\text { 4-class } \\
\text { structure }\end{array}$} & Cluster 4 (n=39) & $1.70(0.09)$ & 6.2 & vla & $1.70(0.06)$ & 3.6 \\
\hline & Cluster $3(n=25)$ & $1.48(0.13)$ & 9 & la & $1.50(0.05)$ & 3 \\
\hline & Cluster $2(n=15)$ & $1.28(0.19)$ & 14.8 & la & $1.25(0.06)$ & 4.8 \\
\hline & Cluster $1(n=11)$ & $1.05(0.18)$ & 17.4 & & $1.06(0.06)$ & 5.7 \\
\hline \multirow{5}{*}{$\begin{array}{c}\text { 5-class } \\
\text { structure }\end{array}$} & Cluster $5(n=27)$ & $1.72(0.07)$ & 3.9 & mod & $1.73(0.04$ & 2.2 \\
\hline & Cluster $4(n=13)$ & $1.65(0.10)$ & 6.2 & la & $1.62(0.02)$ & 1.1 \\
\hline & Cluster $3(n=24)$ & $1.47(0.13)$ & 9 & la & $1.50(0.04)$ & 2.9 \\
\hline & Cluster $2(n=15)$ & $1.28(0.19)$ & 14.8 & la & $1.25(0.06)$ & 4.8 \\
\hline & Cluster $1(n=11)$ & $1.05(0.18)$ & 17.4 & & $1.06(0.06)$ & 5.7 \\
\hline \multirow{6}{*}{$\begin{array}{c}\text { 6-class } \\
\text { structure }\end{array}$} & Cluster $6(n=27)$ & $1.72(0.07)$ & 3.9 & $\bmod$ & $1.73(0.04)$ & 2.2 \\
\hline & Cluster $5(n=13)$ & $1.65(0.10)$ & 6.2 & la & $1.62(0.02)$ & 1.1 \\
\hline & Cluster $4(n=23)$ & $1.48(0.13)$ & 8.8 & la & $1.50(0.04)$ & 2.4 \\
\hline & Cluster $3(n=16)$ & $1.28(0.18)$ & 14.3 & $\bmod$ & $1.26(0.07)$ & 5.2 \\
\hline & Cluster $2(n=7)$ & $1.13(0.16)$ & 13.9 & la & $1.10(0.03)$ & 2.3 \\
\hline & Cluster $1(n=4)$ & $0.90(0.12)$ & 13.2 & & $0.99(0.02)$ & 1.8 \\
\hline \multirow{7}{*}{$\begin{array}{l}\text { 7-class } \\
\text { structure }\end{array}$} & Cluster $7(n=27)$ & $1.72(0.07)$ & 3.9 & $\bmod$ & $1.73(0.04)$ & 2.2 \\
\hline & Cluster $6(n=13)$ & $1.65(0.10)$ & 6.2 & la & $1.62(0.02)$ & 1.1 \\
\hline & Cluster $5(n=23)$ & $1.48(0.13)$ & 8.8 & la & $1.50(0.04)$ & 2.4 \\
\hline & Cluster $4(n=9)$ & $1.37(0.14)$ & 10.4 & la & $1.31(0.04)$ & 3.3 \\
\hline & Cluster $3(n=7)$ & $1.16(0.17)$ & 14.4 & unc & $1.19(0.01)$ & 1.3 \\
\hline & Cluster $2(n=7)$ & $1.13(0.16)$ & 13.9 & la & $1.10(0.03)$ & 2.3 \\
\hline & Cluster $1(n=4)$ & $0.90(0.12)$ & 13.2 & & $0.99(0.02)$ & 1.8 \\
\hline
\end{tabular}

$\mathrm{SD}=$ standard deviation, $\mathrm{CV}$ = coefficient of variation, $\mathrm{ES}=$ effect size, $\mathrm{QO}=$ qualitative outcome, unc = unclear difference, mod $=$ moderate difference, $l a=$ large difference, vla = very large difference.

This article is protected by copyright. All rights reserved. 


\section{FIGURE LEGENDS}

Figure 1. Prediction plot showing the accuracy and error of the ensemble partial least squares regression to predict personal best $100 \mathrm{~m}$ freestyle performance from limb length variables. $\mathrm{R}^{2}=$ coefficient of determination; RMSE = Root mean square error.

Figure 2. Regression coefficients (A) and variable importance scores (B) of limb length variables for the ensemble partial least squares regression. (A) Data are median, quantiles and range of regression coefficients for limb length variables for 500 Monte Carlo experiments. (B) Data are variable importance scores for limb length variables calculated as the mean regression coefficient divided by the standard deviation of the regression coefficient.

Figure 3. Boxplots showing the median, quartiles and range of (A) predicted $100 \mathrm{~m}$ freestyle performance and (B) residuals for Para swimmers with limb deficiency derived from the ensemble partial least squares regression.

Figure 4. The (A) four-class, (B) five-class, (C) six-class, and (D) seven-class classification structures that were derived from Para swimmers predicted $100 \mathrm{~m}$ freestyle performance. (Left) Kernel density estimations were used to identify local minima and maxima and establish class cut-off points. (Middle) The fairness of derived classification structures was evaluated by examining differences in performance between adjacent classes and the within-class variation in actual and predicted performances. (Right) Count plots show the types of limb deficiency groups that from within the derived classes.

This article is protected by copyright. All rights reserved. 


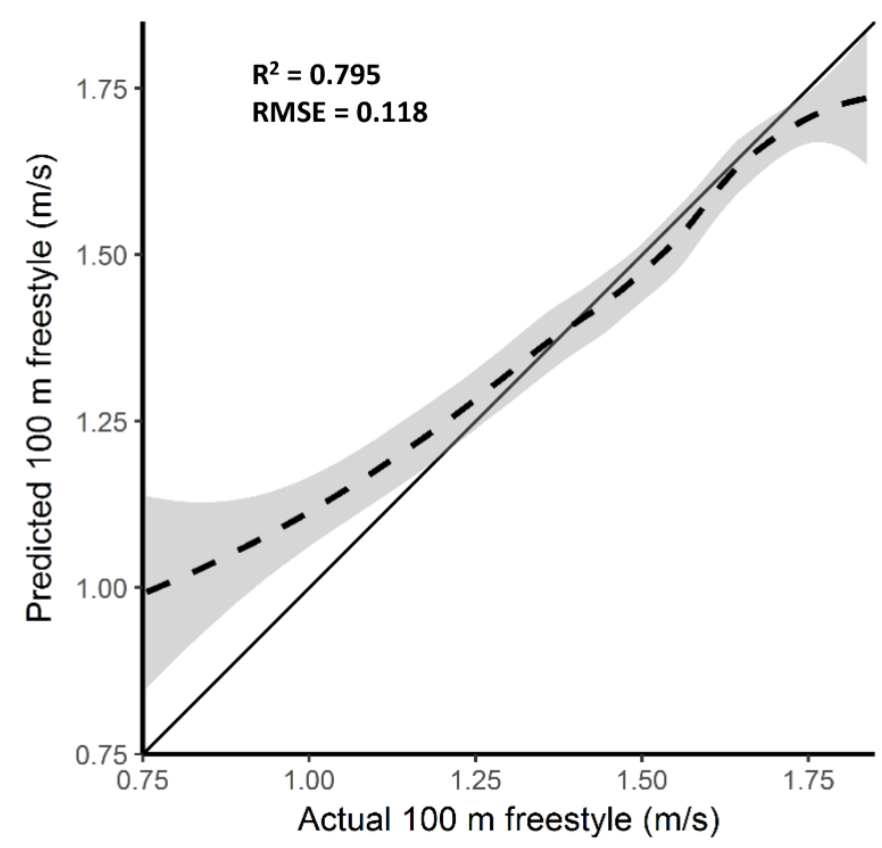

This article is protected by copyright. All rights reserved. 
A

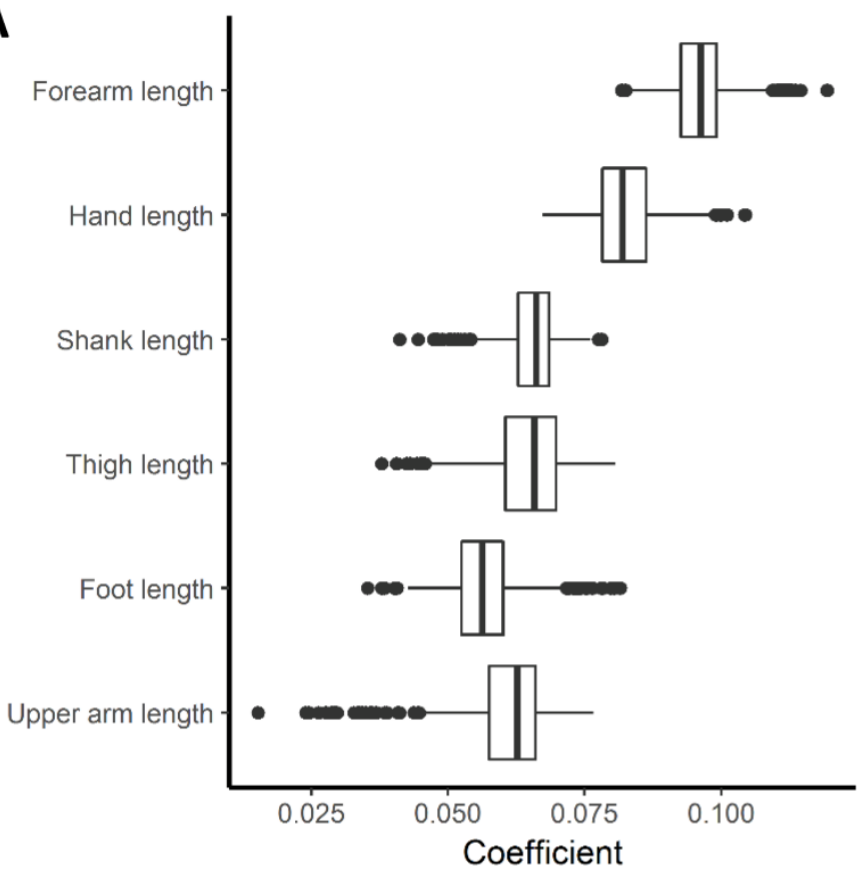

B

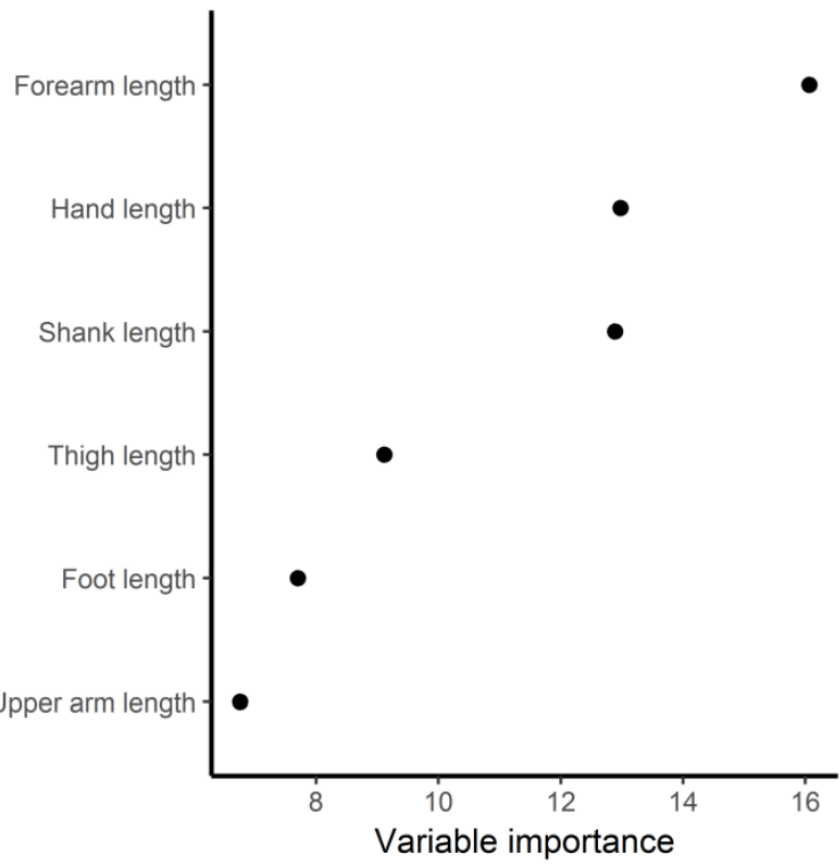

This article is protected by copyright. All rights reserved. 
A

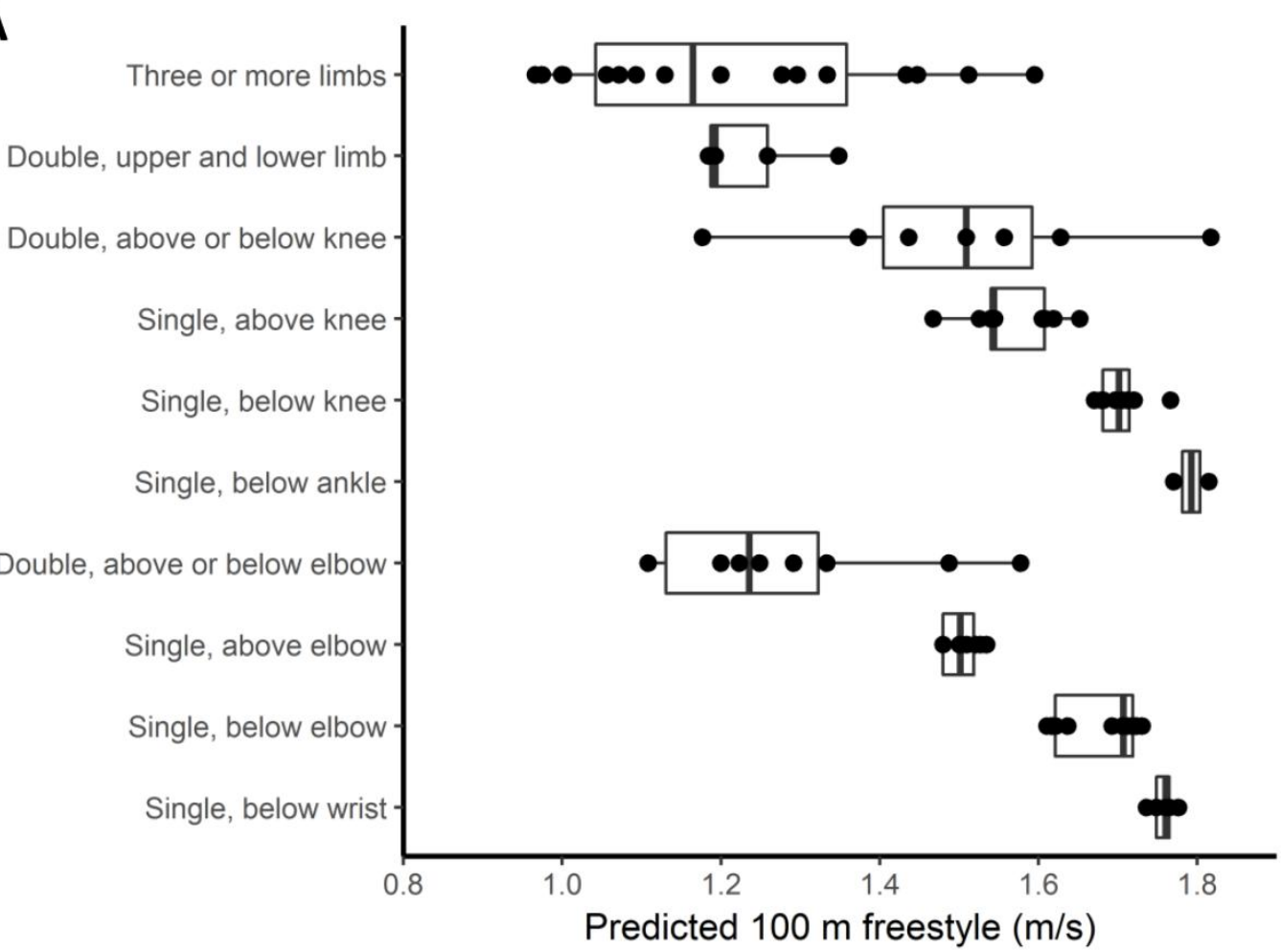

B

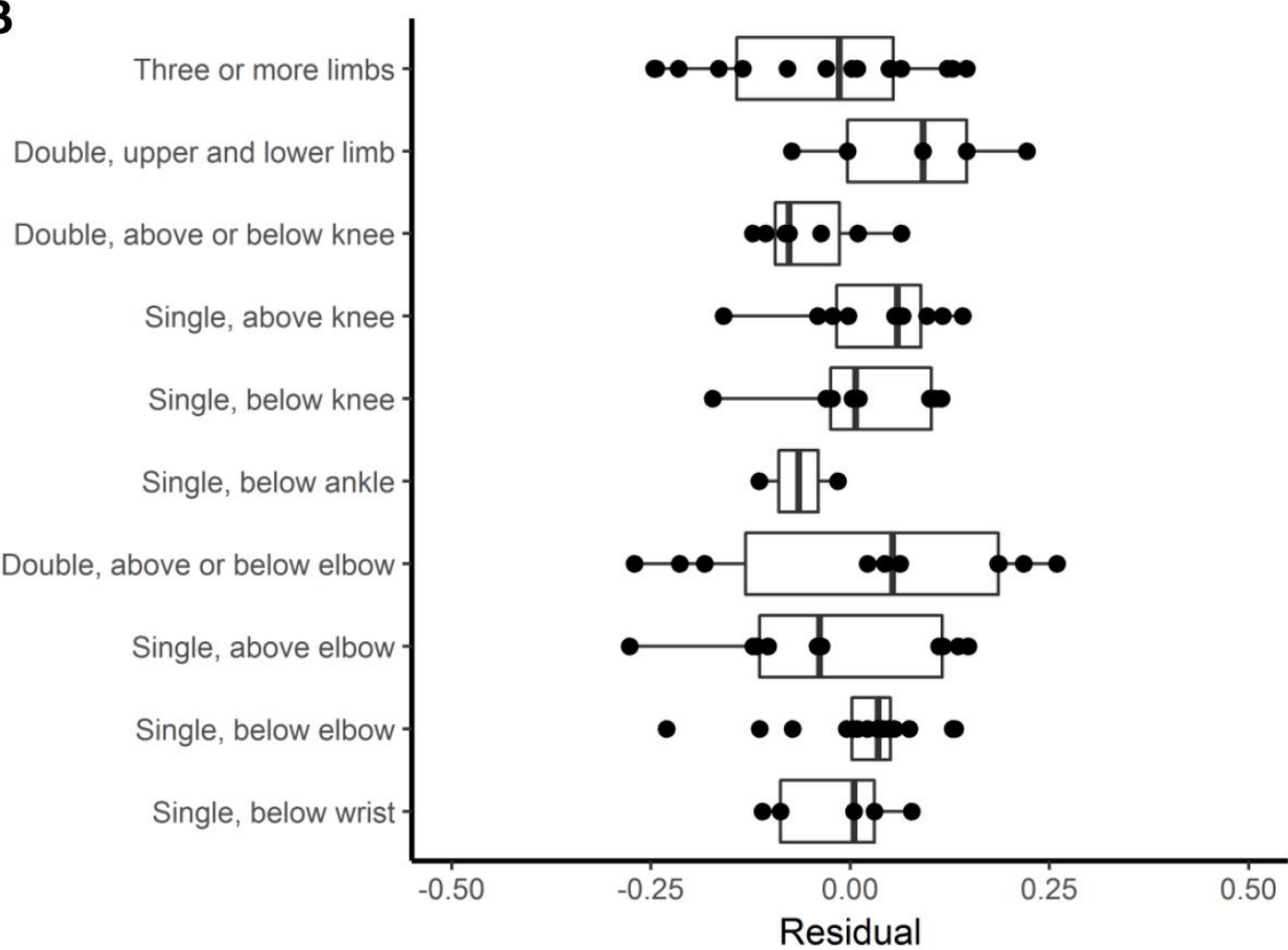

This article is protected by copyright. All rights reserved. 

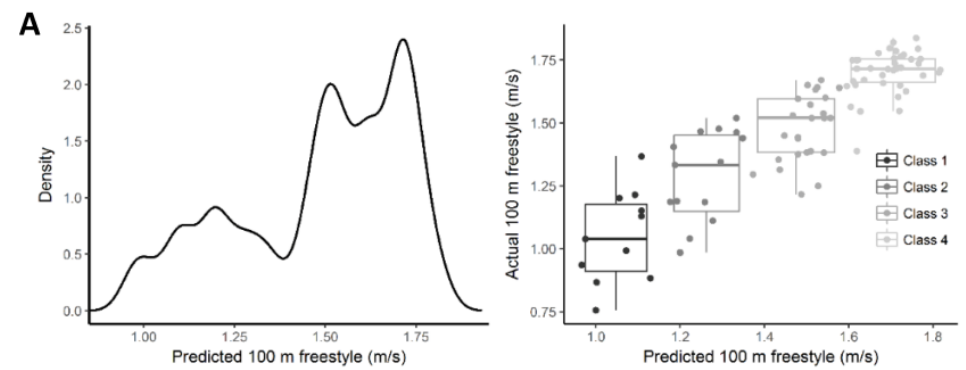

B

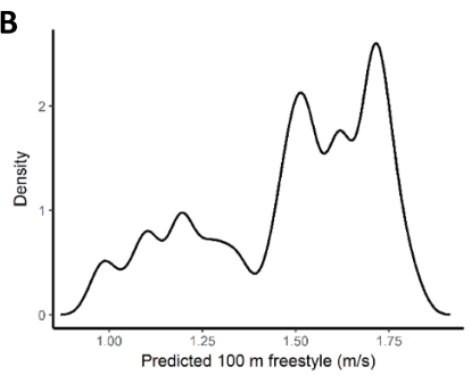

C

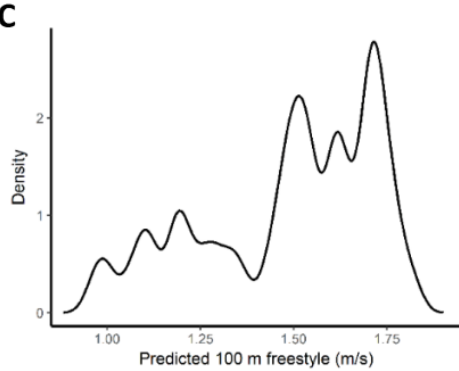

D

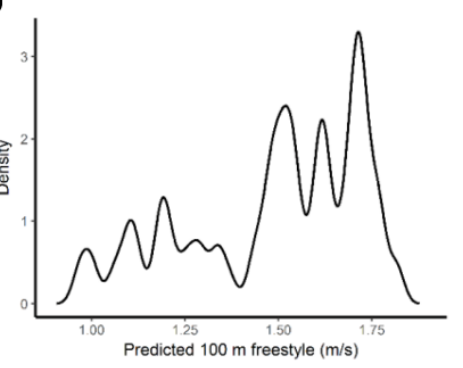

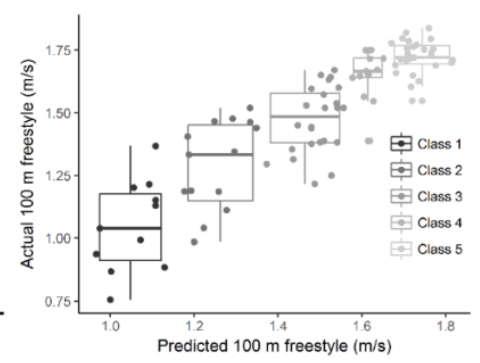
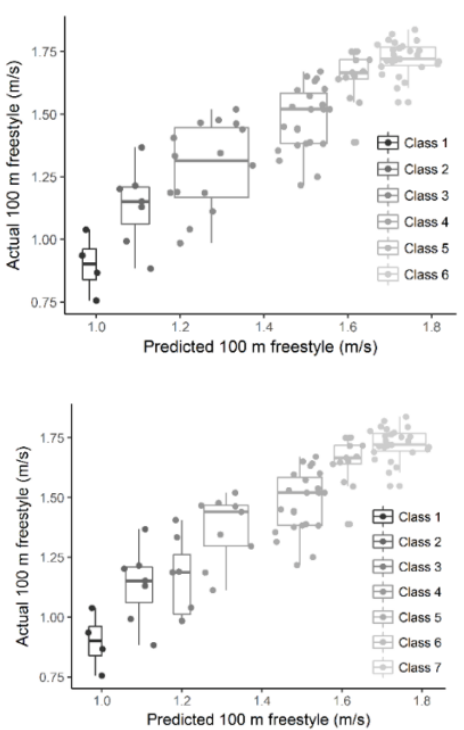
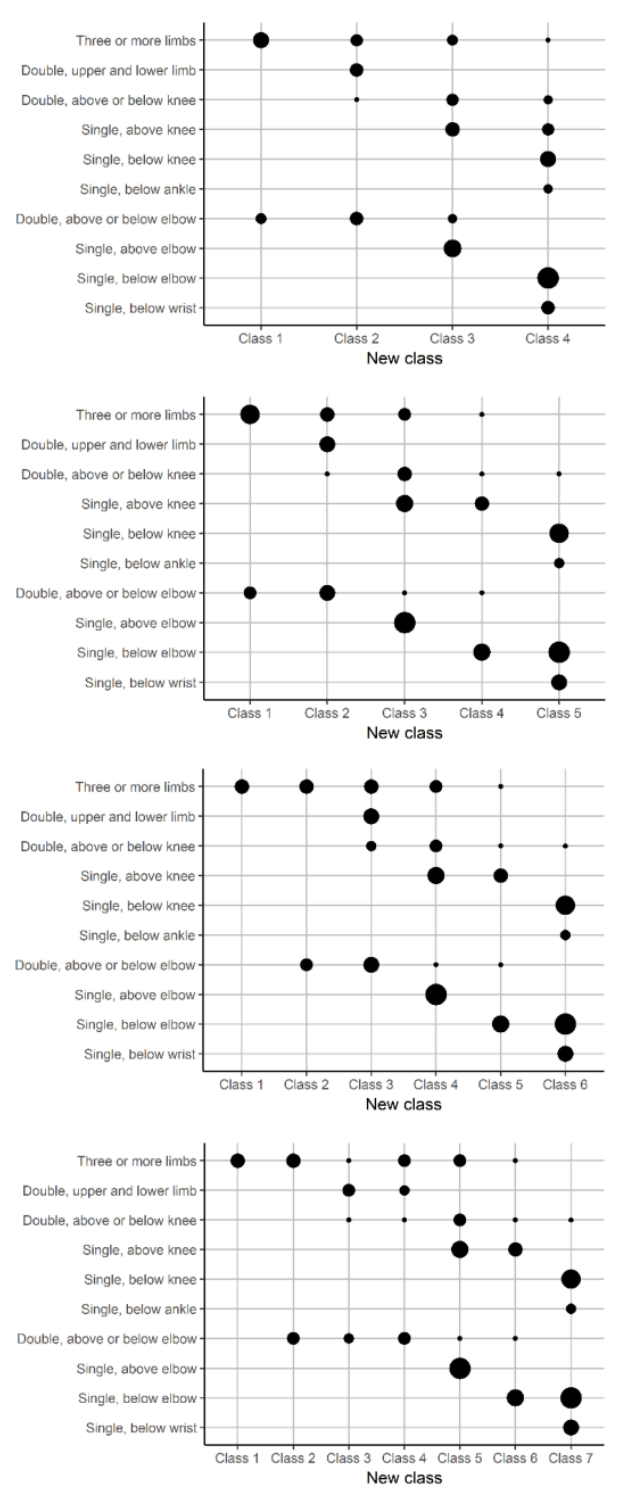

This article is protected by copyright. All rights reserved. 